\title{
Integrated System for Heading Determination
}

\author{
P. KANIEWSKI* AND J. KAZUBEK
}

Military University of Technology, Gen. S. Kaliskiego 2, 00-908 Warsaw, Poland

\begin{abstract}
The paper presents a design and a prototype of integrated system, designed for heading determination of land vehicles. The heading is an angle between the North direction (true or magnetic) and the direction of longitudal axis of a vehicle in the horizontal plane. In land vehicle positioning and navigation, for most of the time the heading can be considered the same as the direction of movement of the vehicle. Thus, it can be used, along with information about the linear displacement of the vehicle, for dead reckoning, i.e. calculation of current vehicle position by accumulating position increments from a known initial location. The determination of vehicle heading represents a key function of dead reckoning and significantly influences the accuracy of land vehicle positioning. Each of the angular sensors, commonly used for heading determination, has serious drawbacks and joint processing of data from several devices can be advantageous. The paper contains a short description of operation, properties and error characteristics of gyros and electronic compasses, which are frequently applied in heading determination application. Further part of the paper presents a design of integrated system, composed of the above mentioned devices. The system processes navigation data with use of a single complementary Kalman filter. A prototype of the designed system has been constructed and tested by the authors. The paper contains a detailed description of the prototype and chosen results of its testing.
\end{abstract}

PACS numbers: 42.81.Pa, 85.70.kh, 85.85.+j, 89.40.Bb

\section{Introduction}

The dead reckoning (DR) is a relative method of positioning, which consists in measuring distance increments travelled by a land vehicle in short time intervals $T$ and accumulating position changes along the axes of chosen frame of reference, with respect to a known initial location [1].

To calculate position changes, the directions of distance increments have to be measured and taken into account [1]. In many land navigation applications, only horizontal position of the vehicle is of interest. A two-dimensional position of vehicle can be expressed with a pair of coordinates $(E, N)$ in a Cartesian horizontal frame of reference OEN, whose axes coincide with the local East and North directions at the chosen point of origin $\mathrm{O}$. The idea of dead reckoning in OEN frame is explained in Fig. 1.

One of the data streams needed for DR can be provided by a heading reference system (HRS). HRS provides estimates of heading $\psi$, which allow DR to calculate the direction of vehicle displacement and predict its current position. Such an HRS system, designed and constructed by the authors, is a subject of this paper.

\section{Heading sensors}

Commonly used heading sensors include difference odometers, gyros and electronic magnetic compasses as

\footnotetext{
* corresponding author; e-mail: pkaniewski@wel.wat.edu.pl
}



Fig. 1. Principle of dead reckoning.

well as GNSS receivers [2-5]. The designed HRS system utilizes two of the above mentioned sensors, i.e. an electronic compass and a gyro. The main subsystem of HRS is an electronic compass module. This part of the system allows heading calculation, based on the Earth magnetic field measurements. Since the Earth magnetic and geographic poles do not coincide, the heading calculated on the basis of magnetic field measurements is called the magnetic heading. To calculate the true heading, i.e. the heading referenced to geographical North direction, a magnetic declination has to be subtracted from the magnetic heading.

To determine the magnetic heading, the components of Earth's magnetic field have to be measured in two, parallel to the Earth surface, orthogonal directions. The Earth magnetic field vector $\boldsymbol{H}$ and its components are shown in Fig. $2[2,6]$. 


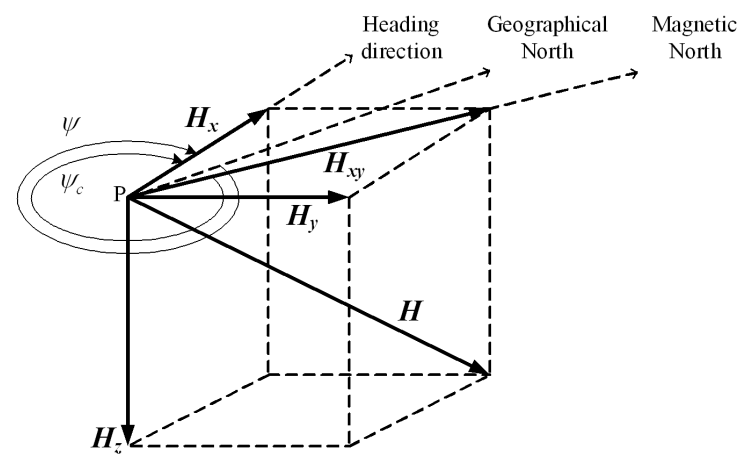

Fig. 2. Earth's magnetic field vector and its components.

The Earth magnetic field vector can be decomposed into a vertical component $\boldsymbol{H}_{z}$ and a horizontal component $\boldsymbol{H}_{x y}$. The direction of the $\boldsymbol{H}_{x y}$ vector is parallel to the magnetic South-North direction. Next, the horizontal component vector $\boldsymbol{H}_{x y}$ can be shown as a sum of two vectors: $\boldsymbol{H}_{x}$, which is parallel to the longitudal axis of the vehicle (assumed the same as the movement direction) and $\boldsymbol{H}_{y}$, which is parallel to the lateral axis of the vehicle and perpendicular to $\boldsymbol{H}_{x}$. It has been assumed that the positive senses of these components correspond to the forward and right directions of longitudal and lateral axes of the vehicle. As can be seen from Fig. 2, the magnetic heading can be calculated as follows $[2,6]$ :

$$
\psi_{\mathrm{c}}=2 \pi-\operatorname{atan}\left(H_{y} / H_{x}\right) .
$$

To calculate the heading with use of the above equation, it is sufficient to measure only values of two horizontal components of the Earth magnetic field, i.e. $H_{x}$ and $H_{y}$. As it is crucial to measure the horizontal components of magnetic field, the magnetic sensors in the compass module should be gimballed mechanically or electronically.

An application of electronic compasses in land vehicles poses serious problems with their installation and calibration. Magnetic disturbances from metal parts of the vehicle and its load, as well as from nearby objects passed by the vehicle may seriously affect the accuracy of measurements. The advantage of compass consists in its bounded errors, not increasing with the time of operation or the distance travelled by the vehicle.

Another type of angular motion sensor is the gyro [7-9]. A gyro may have an analogue or digital interface. The former outputs a continuous voltage $u(t)$, proportional to the angular velocity $\omega(t)$, whereas the latter provides for discrete angular increments $\Delta \psi_{\mathrm{g}}(k)$ around its sensitivity axis. The angular velocity sensed by the vertical gyro includes also components resulting from the Earth rotation and from the vehicle motion, and can be expressed as follows [7]:

$$
\omega_{\mathrm{g}}=\omega-\Omega \sin \varphi-\frac{\mathrm{d} \lambda}{\mathrm{d} t} \sin \varphi,
$$

where $\Omega$ is the Earth rotation rate, whereas $\varphi$ and $\lambda$ are geographic coordinates of the vehicle.
In low-cost micro-electro-mechanical system (MEMS) gyros $[7,8]$, with drifts significantly exceeding the magnitudes of the second and the third term of the above equation, these additional terms can be omitted, which leads to the following simplification: $\omega_{\mathrm{g}} \approx \omega$.

One should also note that in case of deflection of the vehicle vertical axis from the normal to the Earth ellipsoid, the angular velocity sensed by the gyro will be smaller than the true one, and it can be corrected with the following formulae [4]:

$$
\omega=\omega_{\mathrm{g}} / \sqrt{1-\theta^{2}-\phi^{2}},
$$

which holds for small angles of pitch $\theta$ and roll $\phi$. Use of this correction, however, requires that additional inclination sensors should be installed in the system.

The gyro used in the designed HRS system has a digital interface. The heading $\psi_{\mathrm{g}}(n)$ at the time $n T$ can be calculated by counting angular increments $\Delta \psi_{\mathrm{g}}(k)$ from the gyro, which has its sensitivity axis aligned with the vertical axis of the vehicle. The initial heading $\psi(0)$ has to be known and the following formulae can be applied for heading calculations:

$$
\psi_{\mathrm{g}}(n)=\psi(0)+\sum_{k=1}^{n} \Delta \psi_{\mathrm{g}}(k) .
$$

Use of gyros for heading determination has a significant disadvantage, because its errors accumulate along with the time of operation and depend on the shape of vehicle trajectory $[10,7]$. On the other hand, installation of a gyro in a vehicle is relatively simple and prices of accurate FOG and RLG sensors [7, 11] are becoming attractive nowadays. Also new MEMS sensors are becoming still more and more accurate and in many applications they can be an attractive alternative for significantly more expensive FOG or RLG gyros.

\section{Design and operation of HRS}

A block scheme of designed HRS is shown in Fig. 3. The scheme contains sensors and the elements of data processing algorithm, implemented in the system. As can be seen, the HRS system processes gyroscope data and magnetic sensors data to determine a current heading of the vehicle.

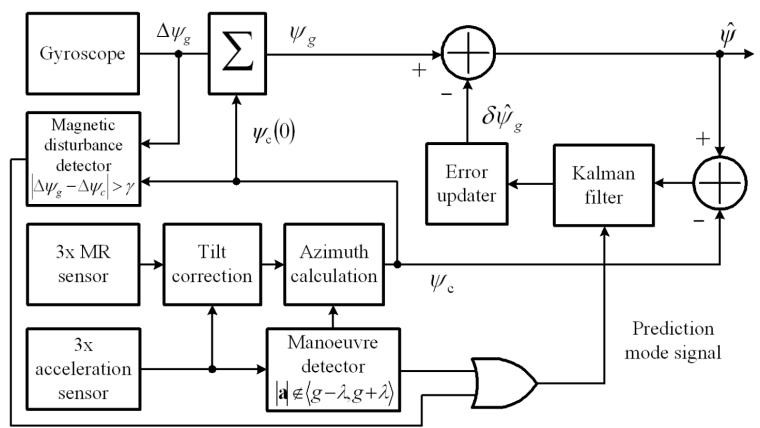

Fig. 3. Block scheme of heading reference system. 
Among many different types of magnetic field sensors available nowadays, magnetoresistive (MR) sensors are an interesting choice for use in low-cost electronic compasses. Their main advantages, besides their cost, are high sensitivity and a large scale of integration, which allows reduction of their dimensions in comparison to formerly widely used fluxgate sensors. In the designed HRS system, three Philips KMZ51 [12] MR sensors have been applied.

It should be noted that when the compass module is tilted, the $H_{x}$ and $H_{y}$ components of magnetic field measured by the MR sensors do not equal horizontal components of the Earth magnetic field [2,3]. Thus, the heading calculated with use of only two magnetic sensors is sensitive to the tilt of compass and it cannot be used if the system has to operate properly in land vehicle moving on non-flat surfaces.

The heading error $\varepsilon$ influenced by tilting compass module can be calculated as [6]:

$$
\varepsilon=\operatorname{atan}(\tan \delta \sin \tau),
$$

where $\tau$ is the tilt angle and $\delta$ is the magnetic inclination angle. How large is this error can be best demonstrated with an example. The inclination angle in Warsaw, Poland equals $c a .68^{\circ}$, which even for a moderate tilt angle of $10^{\circ}$ results in significant heading error exceeding $23^{\circ}$.

To reduce this error, electronic tilt compensation has been applied in the designed HRS. The compensation is realized by transformation of magnetic field measurements from three orthogonal MR sensors from the frame of reference $O X_{\mathrm{C}} Y_{\mathrm{C}} Z_{\mathrm{C}}$ aligned with the compass module to a frame $O X_{\mathrm{E}} Y_{\mathrm{E}} Z_{\mathrm{E}}$ which has its $O X_{\mathrm{E}} Y_{\mathrm{E}}$ plane perpendicular to the local gravity vector. The values of magnetic field horizontal components in $O X_{\mathrm{E}} Y_{\mathrm{E}}$ plane can be calculated as follows $[2,3,5]$ :

$$
\begin{aligned}
& H_{x}^{\prime}=H_{x} \cos \theta-H_{y} \sin \theta \sin \phi-H_{z} \sin \theta \cos \phi, \\
& H_{y}^{\prime}=H_{y} \cos \phi+H_{z} \sin \phi .
\end{aligned}
$$

Having calculated transformed $H_{x}^{\prime}$ and $H_{y}^{\prime}$ magnetic field components, tilt compensated heading can be determined as follows:

$$
\psi_{\mathrm{c}}=2 \pi-\operatorname{atan}\left(H_{y}^{\prime} / H_{x}^{\prime}\right) \text {. }
$$

To evaluate the above equations, the vertical component of the Earth magnetic field $H_{z}$ as well as pitch $\theta$ and roll $\phi$ angles of the module have to be measured. The vertical component is measured with a third MR sensor and tilt angles are calculated with use of a 3-axis MEMS accelerometer MMA7260 by Freescale [11]. The accelerometer is applied to measure the Earth gravity acceleration components $a_{x}, a_{y}, a_{z}$ along its three orthogonal axes. The pitch and roll angles are calculated according to the following formulae $[5,6]$ :

$$
\begin{aligned}
& \theta=\operatorname{atan}\left(a_{x} / \sqrt{a_{y}^{2}+a_{z}^{2}}\right), \\
& \phi=\operatorname{atan}\left(a_{y} / \sqrt{a_{x}^{2}+a_{z}^{2}}\right) .
\end{aligned}
$$

The compass heading $\psi_{\mathrm{c}}$ is used by the Kalman filter only if it has been previously properly compensated for tilt effects. This compensation is not possible when the HRS user accelerates, because the accelerometers cannot distinguish user accelerations from the gravity acceleration. Therefore, a manoeuvre detector has been applied in the system. If the total acceleration differs from $1 \mathrm{~g}$ by more than a predefined threshold $\lambda$, the Kalman filter switches to the prediction mode of operation and does not perform the measurement update.

The HRS contains also a one-axis gyroscope sensor. This sensor provides information about the angular rate and the angle increments in short time spans. Its sensitivity axis is aligned with the vertical axis $Z$ of the compass module. In the design, Analog Devices ADIS16251 integrated digital MEMS gyroscope has been used [8]. The algorithm of data processing in HRS checks if there is a consent between the change of gyroscope heading $\Delta \psi_{\mathrm{g}}$ and the change of compass heading $\Delta \psi_{\mathrm{c}}$. If there is a discrepancy between the two values, the algorithm declares a magnetic field disturbance, and switches the Kalman filter to the prediction mode. This allows one eliminating invalid compass data from the filter input.

To determine the gyroscope heading $\psi_{\mathrm{g}}$, angular rate measurements $\omega_{\mathrm{g}}$ are internally integrated in the gyro and the obtained angle increments $\Delta \psi_{\mathrm{g}}$ are accumulated in HRS microcontroller. Unfortunately, these operations lead to accumulation of errors, with the rate depending on the quality of sensor. Moreover, heading calculated on the basis of gyroscope measurements has to be initialized. In the designed system, the initialization is performed during the start-up procedure of the device and $\psi_{\mathrm{g}}(0)$ is set to the initial compass heading $\psi_{\mathrm{c}}(0)$.

The gyro and compass data are jointly processed with use of a single complementary Kalman filter (KF) [10, 9, 13-15]. The HRS system is integrated according to the scheme of compensation with feed-backward correction of estimated gyro errors. The filter processes differences between compass and corrected gyro measurements. As they both are composed of the true heading and respective errors, the differences between the gyro and compass headings consist of combinations of gyro and compass errors. The Kalman filter estimates residual gyroscope heading errors and provides them to the input of the error updater. The error updater accumulates them and calculates the total estimated heading error $\delta \hat{\psi}_{\mathrm{g}}$, which is subsequently subtracted from the gyro heading $\psi_{\mathrm{g}}$, finally providing the estimated heading $\hat{\psi}$.

\section{Kalman filter for HRS}

The Kalman filter for the designed system is based on a discrete HRS model, which is composed of a difference equation of dynamics and an algebraic equation of observation $[10,13,16]$ :

$$
\begin{aligned}
& \boldsymbol{x}(k+1)=\boldsymbol{\Phi}(k+1, k) \boldsymbol{x}(k)+\boldsymbol{w}(k)-\delta \hat{\boldsymbol{x}}(k), \\
& \boldsymbol{z}(k)=\boldsymbol{H}(k) \boldsymbol{x}(k)+\boldsymbol{v}(k),
\end{aligned}
$$


tor of random process disturbances, $\delta \hat{\boldsymbol{x}}$ - vector of deterministic inputs (corrections from the KF and error updater), $\boldsymbol{\Phi}$ - state transition matrix, $\boldsymbol{z}$ - measurement vector, $\boldsymbol{v}$ - vector of measurement noises, $\boldsymbol{H}$ - observation (measurement) matrix. The first equation of the above discrete model has been formulated based on the following continuous model of heading errors $[7,9,17]$ :

$$
\begin{aligned}
& \delta \dot{\psi}_{\mathrm{g}}=\delta k \omega+b+u_{\psi}, \\
& \delta \dot{k}=u_{k}, \\
& \dot{b}=u_{b},
\end{aligned}
$$

where $\delta \psi_{\mathrm{g}}$ - gyro heading error, $\delta k$ - scale factor error, $\omega$ - angular velocity of the vehicle around the gyro sensitivity axis, $b$ - gyro bias, $u_{\psi}, u_{k}, u_{b}$ - forcing functions (Gaussian white noises with power spectral densities $S_{\psi}$, $\left.S_{k}, S_{b}\right)$. The above scalar equations can be grouped into one matrix equation of the following form:

$$
\frac{\mathrm{d}}{\mathrm{d} t}\left[\begin{array}{c}
\delta \psi_{\mathrm{g}} \\
\delta k \\
b
\end{array}\right]=\left[\begin{array}{lll}
0 & \omega & 1 \\
0 & 0 & 0 \\
0 & 0 & 0
\end{array}\right]\left[\begin{array}{c}
\delta \psi_{\mathrm{g}} \\
\delta k \\
b
\end{array}\right]+\left[\begin{array}{l}
u_{\psi} \\
u_{k} \\
u_{b}
\end{array}\right] .
$$

The discrete equivalent of the above equation can be obtained applying methods described in literature, e.g. $[10,7,9,18]$. Taking into account the presence of deterministic inputs in the system, the difference equation of the HRS model is as follows:

$$
\begin{aligned}
& {\left[\begin{array}{c}
\delta \psi_{\mathrm{g}}(k+1) \\
\delta k(k+1) \\
b(k+1)
\end{array}\right]=\left[\begin{array}{ccc}
1 & \Delta \psi_{\mathrm{g}} & T \\
0 & 1 & 0 \\
0 & 0 & 1
\end{array}\right]\left[\begin{array}{c}
\delta \psi_{\mathrm{g}}(k) \\
\delta k(k) \\
b(k)
\end{array}\right]} \\
& -\left[\begin{array}{c}
\delta \hat{\psi}_{\mathrm{g}}(k) \\
0 \\
0
\end{array}\right]
\end{aligned}
$$

and the state transition matrix $\boldsymbol{\Phi}$, necessary for KF implementation has the following form:

$$
\boldsymbol{\Phi}=\left[\begin{array}{ccc}
1 & \Delta \psi_{\mathrm{g}} & T \\
0 & 1 & 0 \\
0 & 0 & 1
\end{array}\right],
$$

where $\Delta \psi_{\mathrm{g}}$ represents a change of heading between times $k T$ and $(k-1) T$, as reported by gyro.

The other matrix which has to be known and is used by the $\mathrm{KF}$ is the covariance matrix of random process disturbances $\boldsymbol{Q}$. It has been calculated with use of transfer function method [10] and is as follows:

$$
\begin{gathered}
\boldsymbol{Q}= \\
{\left[\begin{array}{ccc}
T S_{\psi}+T \Delta \psi_{\mathrm{g}}^{2} S_{k} / 3+T^{3} S_{b} / 3 & \Delta \psi_{\mathrm{g}} T S_{k} / 2 & T^{2} S_{b} / 2 \\
\Delta \psi_{\mathrm{g}} T S_{k} / 2 & T S_{k} & 0 \\
T^{2} S_{b} / 2 & 0 & T S_{b}
\end{array}\right] .}
\end{gathered}
$$

The input of KF in the HRS system represents a difference between the corrected gyro heading $\hat{\psi}=\psi_{\mathrm{g}}-\delta \hat{\psi}_{\mathrm{g}}$ and the heading from the electronic compass $\psi_{\mathrm{c}}$. Treating the correcting term $\delta \hat{\psi}_{\mathrm{g}}$ as a deterministic input signal (a component of the vector of deterministic inputs $\delta \hat{\boldsymbol{x}}$ ), it can be assumed that it represents a part of the dynamics model rather than the observation model.

Thus, the observation model of HRS is given by the following equation, in which $\psi$ represents the true value of heading:

$$
\begin{aligned}
z & =\psi_{\mathrm{g}}-\psi_{\mathrm{c}}=\left(\psi+\delta \psi_{\mathrm{g}}\right)-\left(\psi+v_{\mathrm{c}}\right)=\delta \psi_{\mathrm{g}}-v_{\mathrm{c}} \\
& =\left[\begin{array}{lll}
1 & 0 & 0
\end{array}\right]\left[\begin{array}{c}
\delta \psi_{\mathrm{g}} \\
\delta k \\
b
\end{array}\right]-v_{\mathrm{c}} .
\end{aligned}
$$

The above equation can be compared to the general equation (12) and, as one can see, the measurement vector $\boldsymbol{z}$ and the vector of measurement noises $\boldsymbol{v}$ become scalars $z$ and $-v_{\mathrm{c}}$ in the observation model of the HRS system. From this comparison, the measurement matrix $\boldsymbol{H}$ can also be determined as

$$
\boldsymbol{H}=\left[\begin{array}{lll}
1 & 0 & 0
\end{array}\right] \text {. }
$$

The Kalman filter equations contain additionally the covariance matrix $\boldsymbol{R}$ of measurement noises $\boldsymbol{v}$, which in the designed HRS system is a scalar, equal to the variance $\sigma_{\mathrm{c}}^{2}$ of electronic compass measurement errors $v_{\mathrm{c}}$ :

$$
\boldsymbol{R}=\sigma_{\mathrm{c}}^{2}
$$

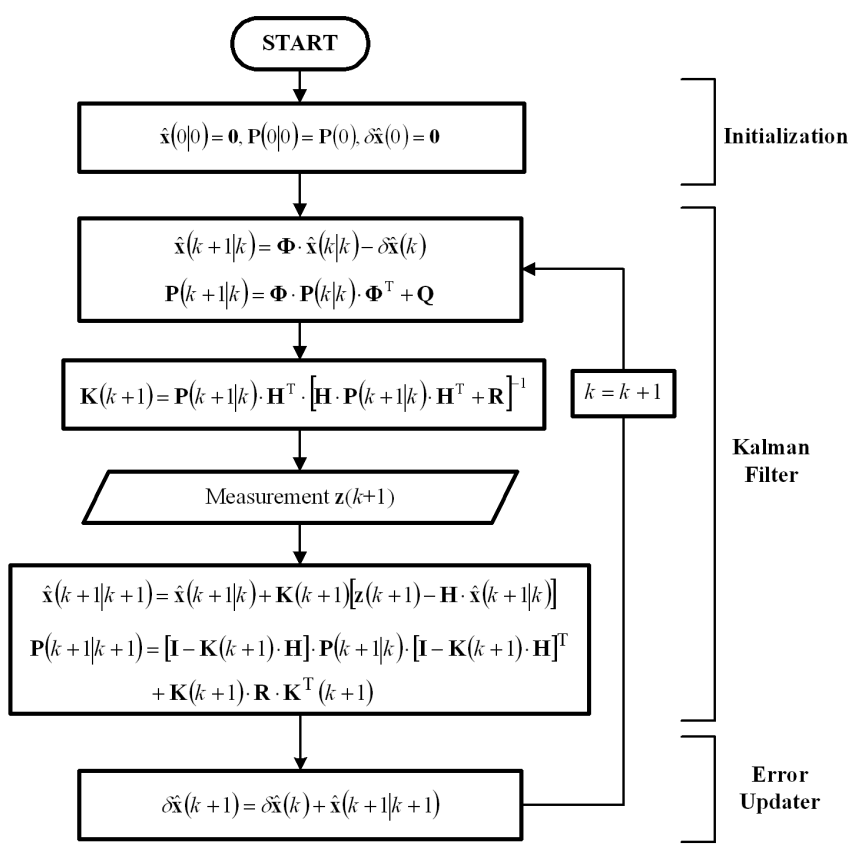

Fig. 4. Kalman filter for heading reference system.

The KF algorithm used in HRS system is composed of initialisation and a series of recursive steps of prediction and correction. The idea of data processing in HRS is presented in Fig. 4. The designations used in this figure and not explained in the text before are as follows: $\hat{\boldsymbol{x}}(k+1 / k+1)$ - predicted state vector at a time $(k+1) T$ - before the measurement update, 
$\hat{\boldsymbol{x}}(k+1 / k+1)$ - filtered state vector at a time $(k+1) T$ - after the measurement update, $\boldsymbol{P}(k+1 \mid k)$ - covariance matrix of prediction errors, $\boldsymbol{P}(k+1 \mid k+1)-$ covariance matrix of filtration errors, $\boldsymbol{K}(k+1)$ - Kalman gains matrix.

\section{Prototype of HRS}

A prototype of the described HRS system has been constructed and tested by the authors of the paper. The main task of the HRS system is to determine the current heading on the almost continuous basis and with possibly high accuracy. It was assumed that the system should be based on low-cost elements, having possibly high accuracy, small dimensions, and be adequate for battery supply. The block diagram of the prototype is shown in Fig. 5.



Fig. 5. Block scheme of HRS prototype.

The HRS is controlled by an Atmel AVR series ATMega644P 8-bit microcontroller, which executes a programme conducting initialization, measurement routines and errors estimation.

The electronic compass module consists of three magnetic field (MR) KMZ51 sensors with orthogonally oriented axes. The outputs of MR sensors are connected to three differential input channels of a 24-bit delta-sigma analogue to digital converter AD7799 from Analog Devices [14]. The $\mathrm{A} / \mathrm{D}$ converter is controlled by the microcontroller through serial peripheral interface (SPI). The conversion results are located in internal registers of AD7799 converter and are read by the microcontroller through the SPI interface.
The re-magnetizing circuit provides short pulses of bidirectional current to the flip coils of MR sensors, which are necessary for so-called flipping of KMZ51 characteristics. The flipping technique is used for measuring and removing voltage offsets of MR sensors, which would otherwise significantly affect the accuracy of magnetic field measurements $[15,16,12]$.

The system uses Freescale 3-axis MMA7260 MEMS accelerometer for determining tilt angles. The accelerometer has got three analogue outputs which drive three low pass filters. The filter outputs are connected to three single-ended inputs of a 10-bit analogue to digital converter, being a part of the microcontroller. The conversion results are available in appropriate registers of the microcontroller.

The gyroscope used in the designed HRS prototype is an Analog Devices ADIS16251 integrated angular rate sensor [8]. The device is controlled by the SPI interface using a set of internal registers. The sensor has an internal integration algorithm providing total angle, which can be used for calculation of angle increments. The HRS takes the advantage of this useful feature.

The estimated heading is displayed on a graphical LCD module with additional information such as pitch and roll angles, current mode of filter operation (only prediction or normal work) and information about detected magnetic field disturbances and manoeuvres. The circuit is powered by a small $3.6 \mathrm{~V} / 600 \mathrm{mAh} \mathrm{NiMH}$ accumulator.

\section{Testing results of HRS}

During the evaluation of HRS performance, gyro heading, compass heading and estimated heading outputs have been observed. Measurement results were sent to a PC through the UART communication port. In Fig. 6, measurement results for $\mathrm{HRS}$, constantly pointing $45^{\circ}$ heading have been shown. The results from Fig. 7 have been obtained for HRS pointing $290^{\circ}$ for the first $60 \mathrm{~s}$ and after that time quickly rotated to $320^{\circ}$.

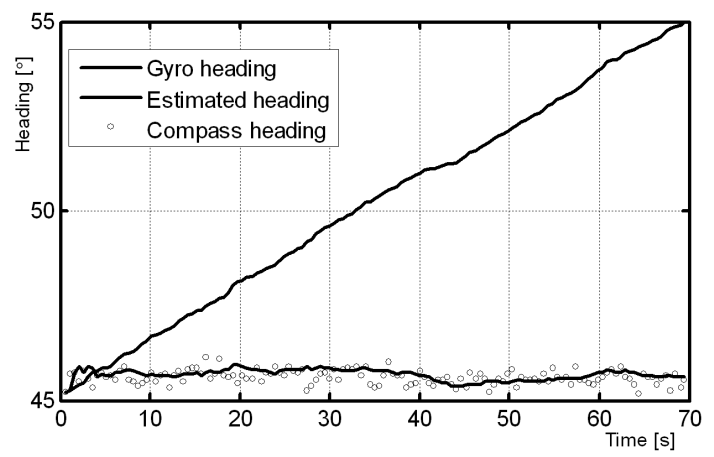

Fig. 6. Headings from gyro, compass and HRS (constant heading scenario).

As can be seen from Figs. 6 and 7, the gyro heading changes even if HRS constantly points at the same direction. This almost linear increase of heading is mainly due 


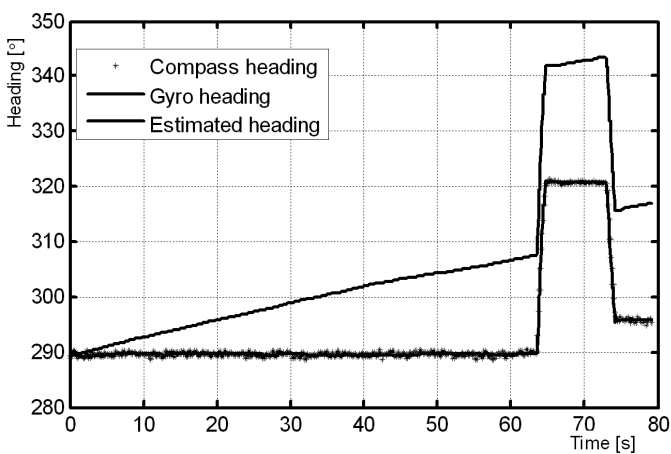

Fig. 7. Headings from gyro, compass and HRS (variable heading scenario).

TABLE

Comparison of compass and HRS heading errors.

\begin{tabular}{l|c|c}
\hline \hline & Compass & HRS \\
\hline standard deviation $[\mathrm{deg}]$ & 0.328 & 0.101 \\
angle error range $[\mathrm{deg}]$ & 1.830 & 0.460
\end{tabular}

to gyro bias. Additional errors appear when the HRS is rotated and this effect is connected with existence of the scale-factor gyro error. The compass heading has long-term stability, but is noisy. The integration algorithm enables estimation of heading, holding the advantages of short-term gyro accuracy and long-term compass stability. Due to use of gyro, fast response of the system has been preserved and noises of the compass heading have been significantly reduced. Simple statistics of errors for the stationary period of time for the second testing scenario (Fig. 7) have been presented in Table.

\section{Acknowledgments}

This work was supported by the Polish Ministry of Science and Higher Education from sources for science in the years 2007-2010 under Commissioned Research Project PBZ-MNiSW-DBO-04/I/2007.

\section{References}

[1] Y. Zhao, Vehicle Location and Navigation Systems, Artech House, Inc., USA 1997.

[2] M.J. Caruso, Applications of Magnetic Sensors for Low Cost Compass Systems, PLANS, London 2000, p. 177.

[3] Q. Ladetto, V. Gabaglio, B. Merminod, Combining Gyroscopes, Magnetic Compass and GPS for Pedestrian Navigation, KIS, Banff, Canada 2001, p. 205.

[4] J. Stephen, G. Lachapelle, J. Navigation 54, 297 (2001).

[5] Electronic Tilt Compensation, Application Note AN00MM-004, Memsic 2008.

[6] Electronic Compass Design using KMZ51 and KMZ52, Application Note AN00022, Philips Semiconductors, 2000.

[7] M.S. Grewal, L.R. Weill, A.P. Andrews, Global Positioning Systems, Inertial Navigation, and Integration 2nd ed., Wiley, New York 2007.

[8] Programmable Low Power Gyroscope ADIS16251, Datasheet, Analog Devices, 2007.

[9] J.A. Farrell, Aided Navigation GPS with High Rate Sensors, McGraw-Hill, USA 2008.

[10] R.G. Brown, P.Y.C. Hwang, Introduction to Random Signals and Applied Kalman Filtering, Wiley, UK 1997.

[11] Micromachined accelerometer MMA7260QT, Freescale Technical Data, 2008.

[12] KMZ51 Magnetic Field Sensor, Data Sheet, Philips Semiconductors, 2000.

[13] Kalman Filtering and Neural Networks, Ed. S. Haykin, Wiley, USA 2001.

[14] 3-Channel ADC ADr799, Datasheet, Analog Devices, 2005.

[15] Set/Reset Function for Magnetic Sensors, Application Note AN213, Honeywell, 2002.

[16] General Magnetoresistive Sensors for Magnetic Field Measurement, Philips Semiconductors, 2000.

[17] K. Barczak, T. Pustelny, D. Dorosz, J. Dorosz, Acta Phys. Pol. A 114, 36 (2008).

[18] A. Szpakowski, T. Pustelny, J. Phys. IV (France) 137, 153 (2006). 